УДК 616.831-001

DOI: $10.26435 /$ UC.V0I3(32).335

\author{
С.Е. Золотухин, А.Д. Есаулов, А.И. Фабер
}

ГОО ВПО «Донецкий национальный медицинский университет имени М. Горького», Донецк

\title{
ОСОБЕННОСТИ ИЗМЕНЕНИЯ ОТДЕЛЬНЫХ ПОКАЗАТЕЛЕЙ КАЛЬЦИЕВОГО ОБМЕНА И ЦИТОКИНОВ ПРИ ЛЕЧЕНИИ ОСТЕОПОРЕТИЧЕСКИХ КРЫС С ТЯЖЕЛОЙ МЕХАНИЧЕСКОЙ ТРАВМОЙ МИАКАЛЬЦИКОМ
}

В настоящее время частота и тяжесть механических повреждений, обусловленных факторами цивилизации, не имеют тенденции к снижению [3, 4, 6]. Летальность пострадавших при присоединении к травме шока увеличивается до 80 \% [6]. Особенно неблагоприятно протекает травматическая болезнь у людей, имеющих тяжелые фоновые заболевания, в том числе и остеопороз [1, 5]. Тяжелая механическая травма, полученная на фоне глюкокортикоидного остеопороза, характеризуется высокой летальностью и частотой осложнений $[2,6]$. В настоящее время эффективность кальцитонина у пострадавших с тяжелой механической травмой и сопутствующим остеопорозом не изучены, а механизмы его противошокового действия нуждаются в уточнении.

\section{ЦЕЛЬ ИССЛЕДОВАНИЯ}

Оценить эффективность миакальцика (кальцитонина лосося) по характеру изменения отдельных показателей кальциевого обмена и цитокинов у крыс с глюкокортикоидным остеопорозом и шоковым типом течения посттравматической реакции.

\section{МАТЕРИАЛ И МЕТОДЫ}

Экспериментальные исследования выполнены на 42 белых беспородных крысах, весом 250-300 г. Подготовка животных к эксперименту, травмирование и выведение из эксперимента осуществляли при соблюдении общих требований и положений Европейской Конвенции по защите позвоночных животных, используемых для исследовательских и других целей (Страсбург, 1986).

В исследовании изучали эффективность кальцитонина лосося (фармакологический препарат «Миакальцик», выпускаемый фирмой Novartis Pharma в форме ампульного раствора для инъекций). 1мл препарата содержал $100 \mathrm{ME}$ синтетического кальцитонина лосося (1 ME биологи- ческой активности препарата соответствовал 0.2 мкг активного вещества) [7, 8].

Эффективность кальцитонина лосося при моделируемой травме и остеопорозе изучали по увеличению продолжительности жизни крыс, которых лечили препаратом (опытная группа), по сравнению со временем жизни крыс без лечения (группа контроля). Препарат вводили через 4 часа после нанесения травмы и типирования посттравматической реакции. В опытной группе крыс кальцитонин лосося вводили внутрибрюшинно в дозе $25 \mathrm{ME} /$ кг (в объемном эквиваленте это составляло 0,4 мл/кг официнального раствора).

Эффективность кальцитонина лосося при глюкокортикоидном остеопорозе и травме изучали также по изменению комплекса биохимических показателей крови в двух группах крыс, которым вводили препарат (опытная группа 2) и крыс с остеопорозом и травмой без введения препарата (контрольная группа 2). В каждой группе животных было по 8 особей. 10 крыс использованы для сравнения (контрольинтактные животные).

Тяжелую механическую травму моделировали путем нанесения серии из 20 ударов электрическим ударником по каждому бедру крыс силой 250 H/см2 (модификация метода Кеннона). У животных в ответ на механическую травму, стандартную по силе воздействия, развивалась посттравматическая реакция, при которой продолжительность жизни животных колебалась в широких пределах: от полусуток до 5 суток. Большой разброс данных времени жизни крыс вызвал необходимость учета роли индивидуальной реактивности животных. Для этого была применена методика, связанная с измерением электрокожного сопротивления у травмированных крыс [3, 4].

( С.Е. Золотухин, А.Д. Есаулов, А.И. Фабер, 2019 (c) Университетская Клиника, 2019 
Типы посттравматической реакции определяли с помощью модифицированного метода измерения электропроводности кожи посредством показателя «К» в динамике по С.Е. Золотухину [4]. В развитии посттравматической реакции на основании значений показателя «К» дифференцировали три ее основных типа. Первый тип автором методики был назван «шоковым смертельным» [3]. У крыс с этим типом развивался травматический шок, подтверждаемый данными величины АД, ЧСС и частоты дыхания. Продолжительность жизни животных не превышала суток $(18,2 \pm 3,1$ час). Шоковый смертельный тип обычно регистрировали с частотой 40 $\%$. Второй тип посттравматической реакции назван «шоковым несмертельным». Травматический шок у крыс с таким типом развивался, но его тяжесть была меньше по сравнению с группой животных, в которой имел место первый тип реакции. Продолжительность жизни животных с шоковым несмертельным типом посттравматической реакции была в пределах 2-3 суток (60,0 $\pm 5,4$ час). Крысы погибали от развития «шокового легкого» и пневмонии. Частота регистрации животных с указанным типом реагирования составляла $30 \%$. Третий тип реагирования животных на тяжелый механический фактор назван «нешоковым», т.к. шок у животных этой группы не развивался. Продолжительность жизни у травмированных крыс находилась

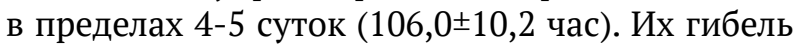
была связана с развитием гнойно-септических осложнений вследствие инфицирования травмированных тканей. Частота развития нешокового типа у животных с моделируемой патологией была равна 30 \% [3].

В настоящем исследовании использованы крысы, у которых дифференцировался только шоковый смертельный тип посттравматической реакции.

Моделирование остеопороза осуществляли путем внутримышечного введения 3 мг/кг метилпреднизолона (фирма «Pfizer», США) в сутки на протяжении 4-5 недель под непосредственным еженедельным рентгенологическим контролем длины пояснично-грудного отдела позвоночника крысы и учета степени уменьшения средней длины одного позвонка этого сегмента, по которой и определяли (посредством вычисления показателя «R») тяжесть остеопороза [2]. В исследовании использованы крысы, у которых развивался остеопороз легкой степени (при более тяжелых формах остеопороза и тяжелой травме имела место ранняя летальность).

При моделировании сочетанной патологии первоначально вызывали у крыс остеопороз, а затем наносили травму.
Определение содержания гормонов (кальцитонина, КТ, паратиреоидного гормона, ПТГ, тиреотропного гормона, ТТГ, тироксина, Т4, и трийодтиронина, Т3, цитокинов (интерлейкина - 6, ИЛ-6) и фактора некроза опухоли - альфа (ФНО- $\alpha)$ проводили иммуноферментным и радиоиммунологическим (метка радиоактивным изотопом I125) методами (с использованием стандартных коммерческих наборов реактивов для определения исследуемых веществ в образцах плазмы крови крыс). Содержание ионизированного кальция в сыворотке крови определяли с помощью ионоселективных электродов на анализаторе «Эксперт 001».

Полученные в результате исследования выборки данных были проверены на соответствие законам нормального (Гаусовского) распределения с применением программного пакета Statistica 5.5 для Windows (StatSoft, США). Табличные данные представлены в виде средней (M) и ее ошибки (m). Достоверность различий между парными выборками данных определялась с применением критерия Стьюдента. Нулевой гипотезой в нашем исследовании мы считали предположение о том, что изучаемые выборки идентичны, а имеющиеся различия - случайны. Пары выборок данных, занесенные в базу данных программы Stadia for DOS, средствами этой программы были проверены на предмет выявления статистически достоверных различий между ними. Различия между парными выборками считались статистически достоверными при вероятности нулевой гипотезы менее $5 \%$. Поскольку все данные в полученных выборках подчинялись законам нормального распределения, непараметрические методы статистического исследования не применялись.

\section{РЕЗУЛЬТАТЫ И ОБСУЖДЕНИЕ}

Продолжительность жизни у остеопоретических (остеопороз легкой степени тяжести) крыс с травмой при развитии шокового смертельного типа посттравматической реакции (контроль 1) была равна $12,2 \pm 2,2$ часа (без лечения). Для сравнения продолжительность жизни крыс с шоковым смертельным типом посттравматической реакции без остеопороза и лечения составила $17,3 \pm 2,2$ час. Продолжительность жизни крыс в опытной серии (с остеопорозом, с травимой и с лечением) составила - 19,7 $\pm 2,3$ часа. Разница по сравнению с соответствующим контролем составила 7,5 часа $(\mathrm{p}<0,05)$. Результаты биохимических исследований у крыс контрольных и опытной серий представлены в таблице.

Как видно из представленных данных, у крыс с шоковым смертельным типом посттравматической реакции и остеопорозом легкой степени 
Изменения биохимических и иммунологических показателей крови у остеопоретических крыс при шоковом смертельном типе посттравматической реакции в сериях экспериментов (M士m)

Таблица.

\begin{tabular}{|c|c|c|c|}
\hline \multirow{2}{*}{ Показатели, ед } & \multirow{2}{*}{$\begin{array}{c}\text { Контроль } \\
\text { (интактные), }(\mathrm{n}=10)\end{array}$} & \multicolumn{2}{|c|}{ Серии эксперимента } \\
\hline & & $\begin{array}{c}\text { Контроль } 2 \\
\text { (без лечения), }(\mathrm{n}=8) \\
\end{array}$ & $\begin{array}{c}\text { Опытная } 2 \\
\text { (миакальцик), }(\mathrm{n}=8) \\
\end{array}$ \\
\hline Са++, мкмоль/л & $0,63 \pm 0,03$ & $0,39 \pm 0,02 *$ & $0,49 \pm 0,01^{* \#}$ \\
\hline КТ, пг/мл & $24,11 \pm 3,1$ & $72,3 \pm 7,2 *$ & $228,7 \pm 6,8 * \#$ \\
\hline ПТГ, пг/мл & $51,25 \pm 6,91$ & $320,4 \pm 26,7^{*}$ & $462,3 \pm 28,4^{*} \#$ \\
\hline ТТГ, мМО/л & $0,129 \pm 0,022$ & $0,57 \pm 0,04^{*}$ & $0,69 \pm 0,03^{*} \#$ \\
\hline Т4, нмоль/л & $39,67 \pm 6,8$ & $120,6 \pm 8,5^{*}$ & $106,2 \pm 9,9 * \#$ \\
\hline Т3, нмоль/л & $2,45 \pm 0,19$ & $89 \pm 0,02 *$ & $2,2 \pm 0,16^{*}$ \\
\hline ИЛ-6, пг/мл & $7,5 \pm 1,6$ & $77,9 \pm 4,0 *$ & $60,3 \pm 4,4^{*} \#$ \\
\hline ФНО- $\alpha$, пг/мл & $44,72 \pm 3,78$ & $1935,3 \pm 134,5^{*}$ & $1188,1 \pm 112,7^{* \#}$ \\
\hline
\end{tabular}

Примечание: * - обозначена достоверность различий показателей по сравнению с группой интактных животных (p<0,05); \# - обозначена достоверность различий показателей по сравнению с группой травмированных крыс без лечения $(\mathrm{p}<0,05)$.

через 8 часов после травмы (контрольная серия 2) наблюдались глубокие нарушения метаболизма Са++, выраженная нейроэндокринная реакция и гиперцитокинемия. Изменения исследованных биохимических показателей в этой серии характеризуют тяжесть и необратимость патологического процесса у животных с шоковым смертельным типом посттравматической реакции и остеопорозом (они касаются, в первую очередь, уровня ионизированного кальция и цитокинов - ИЛ-6 и ФНО- $\alpha$ ).

У животных, которым вводили внутрибрюшинно миакальцик наблюдались изменения, отличающиеся от контроля (травма без лечения). Обращало на себя внимание, что возрастала на $15,8 \%$ (p<0,05) концентрация ионизированного кальция, резко в 3,2 раза увеличивалась концентрация КТ (p<0,05), в 1,4 раза возрастала концентрация ПТГ (p<0,05) и в 1,2 раза концентрация ТТГ (p<0,05). При этом на 36\% снижалась концентрация Т4 (p<0,05). Уровень Т3 не изменялся. Уровень ИЛ-6 снижался в 1,3 раза ( $\mathrm{p}<0,05)$, а ФНО- $\alpha$ в 1,6 раза $(\mathrm{p}<0,05)$. Изменение всех показателей следует считать благоприятными, поскольку у животных с аналогичной тяжестью и дозой миакальцика наблюдалось увеличение продолжительности жизни. Благоприятную картину состояния животных по сравнению с контрольной группой также подчеркивают увеличение концентрации кальция и некоторое снижение цитокиновых показателей. Возросшая в 3,2 раза концентрация КТ говорила об экзогенно введенном кальцитонине (в составе фармпрепарата «миакальцик»), который детектировался используемым аналитическим методом. Увеличение концентрации ПТГ и ТТГ после введения миакальцика крысам с шоковым смертельным типом посттравматической реакции и остеопорозом также следует считать результатом действия миакальцика. Исходя из функционально антагонистических механизмов влияния КТ и ПТГ на кости и кальциевый обмен в целом, можно было бы предполагать, что уровень ПТГ после введения КТ лосося должен снижаться. На самом деле, как видно из таблицы, он повысился, причем повысился вместе с ТТГ. Повышение ПТГ и ТТГ в крови вызвано, вероятно, усилением гипофизарного синтеза и выброса гормонов в кровь. Влияние миакальцика на ЦНС подтверждают литературные источники, в частности данные о наличии в ЦНС специфических рецепторов для связывания кальцитонина лосося [8]. Вероятно, с этими свойствами миакальцика связан и его анальгетический эффект. С увеличением концентрации ТТГ не происходило существенных отклонений в уровне гормонов Т4 и Т3, однако незначительные, хотя и достоверные изменения Т4, свидетельствовали о сдвиге в сторону восстановления функциональной связи гипоталамуса и гипофиза с щитовидной железой.

Данные о снижении уровня гиперцитокинемии при введении крысам миакальцика, вероятно, были вызваны положительным влиянием препарата на желудочно-кишечный тракт. Известно, что миакальцик снижает желудоч- 
ную и экзокринную панкреатическую секрецию. Эти свойства препарата обусловливают его эффективность в лечении острого панкреатита [5]. При травматическом шоке указанные механизмы миакальцика препятствуют неполноценному перевариванию пищи, содержащейся в желудочно-кишечном тракте животных, действию агрессивных соков на слизистую оболочку этого тракта и, как следствие, приводят к уменьшению всасываемости непереваренных (полноценная функция желудочно-кишечного тракта при тяжелой механической травме нарушается) компонентов пищи в кровь. Вероятно, следует считать, что за счет снижения интоксикации организма со стороны токсических продуктов желудочно-кишечного тракта снижался уровень ИЛ-6 и ФНО- $\alpha$.

Таким образом, проведена оценка эффективности кальцитонина лосося при тяжелой механической травме и сопутствующем глюкокортикоидном остеопорозе в эксперименте. Она показала эффективность препарата при шоковом смертельном типе посттравматической реакции и глюкокортикоидном остеопорозе легкой степени. Лечебное действие миакальцика при шоке, вероятно, реализуется за счет механизма его нейротропного действия на ЦНС и, в частности, на гипоталамо-гипофизарную систему. Та- ким путем повышается в 1,4 раза $(\mathrm{p}<0,05)$ и в 1,2 раза $(\mathrm{p}<0,05)$ концентрация в крови ПТГ и ТТГ соответственно. Миакальцик, вероятно, также уменьшает интоксикацию организма токсическими продуктами кишечника. Благодаря этому механизму концентрация ИЛ-6 в крови снижается в 1,3 раза (p<0,05), а концентрация ФНО- $\alpha-$ в 1,6 раза $(\mathrm{p}<0,05)$.

\section{В Ы В О Д Ы}

1. Миакальцик (кальцитонин лосося) обладает противошоковой лечебной эффективностью. У животных с сочетанной патологией (шоковый смертельный тип посттравматической реакции, развивающийся у животных с легкой степенью остеопороза) продолжительность жизни крыс по сравнению с контролем (без лечения) увеличивается на 7,5 часов $(\mathrm{p}<0,05)$.

2. Механизм противошокового действия миакальцика реализуется за счет его нейротропного действия на ЦНС и, в частности, на гипоталамогипофизарную систему, а также за счет ограничения процессов пищеварения, что в условиях тяжелой механической травмы уменьшает проникновение в кровь из желудочно-кишечного тракта токсических продуктов гниения и брожения пищи.

\section{С.Е. Золотухин, А.Д. Есаулов, А.И. Фабер}

ГОО ВПО «Донецкий национальный медицинский университет имени М. Горького», Донецк

\section{ЭФФЕКТИВНОСТЬ МИАКАЛЬЦИКА ПРИ ТЯЖЕЛОЙ МЕХАНИЧЕСКОЙ ТРАВМЕ, Г ЛЮКОКОРТИКОИДНОМ ОСТЕОПОРОЗЕ И У ОСТЕОПОРЕТИЧЕСКИХ КРЫС С ТРАВМОЙ}

Работа посвящена оценке эффективности кальцитонина лосося («Миакальцика») при тяжелой механической травме, при глюкокортикоидном остеопорозе и травме, протекающей на фоне остеопороза. В работе использованы физиологичные, патофизиологические, рентгенологические, биохимические и статистические методы исследования. Эффективность препарата определяли путем сравнения продолжительности жизни животных, имеющих шоковый смертельный тип течения посттравматической реакции, развивающийся при стандартной по силе воздействия травме и при глюкокортикоидном остеопорозе легкой степени тяжести в двух группах животных (которых лечили кальцитонином и не лечили). Эффективность кальцитонина лосося при глюкокортикоидном остеопорозе и травме изучали также по изменению комплекса биохимических показателей крови в двух группах крыс, которым вводи- ли препарат (опытная группа 2) и крыс с остеопорозом и травмой без введения препарата (контрольная группа 2). У животных с сочетанной патологией установлена протвошоковая эффективность миакальцика. Лечебное действие миакальцика при шоке, вероятно, реализуется за счет механизма его нейротропного действия на ЦНС и, в частности, на гипоталамогипофизарную систему. Таким путем повышается в 1,4 раза $(\mathrm{p}<0,05)$ и в 1,2 раза $(\mathrm{p}<0,05)$ концентрация в крови ПТГ и ТТГ соответственно. Миакальцик, вероятно, также уменьшает интоксикацию организма токсическими продуктами кишечника. Благодаря этому механизму концентрация ИЛ-6 в крови снижается в 1,3 раза $(\mathrm{p}<0,05)$, а концентрация ФНО- $\alpha-$ в 1,6 раза $(\mathrm{p}<0,05)$.

Ключевые слова: тяжелая механическая травма, глюкокортикоидный остеопороз, кальцитонин лосося. 


\section{S.E. Zolotukhin, A.D. Esaulov, A.I. Faber}

\section{SEI HPE «M. Gorky Donetsk National Medical University», Donetsk}

\section{EFFICACY OF MYACALCIC IN HEAVY MECHANICAL INJURY, GLUCOCORTICOUS OSTEOPOROSIS AND IN OSTEPORETICAL RATS WITH INJURY}

The work is devoted to assessing the effectiveness of salmon calcitonin ("Miacalcic") in severe mechanical injury, in case of glucocorticoid osteoporosis and injury occurring against the background of osteoporosis. The study used physiological, pathophysiological, X-ray, biochemical, and statistical research methods. The efficacy of the drug was determined by comparing the life expectancy of animals with a shock-type lethal course of a post-traumatic reaction, which develops with a standardized impact and mild glucocorticoid osteoporosis in two groups of animals (calcitonin-treated and untreated). The effectiveness of salmon calcitonin in glucocorticoid osteoporosis and injury was also studied by changing the complex of blood biochemical parameters in two groups of rats that were injected with the preparation (test group 2 ) and rats with osteoporosis and injury without drug ad- ministration (control group 2). In animals with a combined pathology, the protwash efficiency of myacalcics was established. The curative effect of myacalcic in shock is probably realized through the mechanism of its neurotropic action on the central nervous system and, in particular, on the hypothalamic-pituitary system. In this way, the concentration of PTH and TSH in the blood increases 1.4 times $(p<0.05)$ and 1.2 times $(p<0.05)$, respectively. Miacalcium probably also reduces the intoxication of the body with toxic intestinal products. Due to this mechanism, the concentration of IL- 6 in the blood decreases by 1.3 times $(\mathrm{p}<0.05)$, and the concentration of TNF- $\alpha$ - by 1.6 times ( $\mathrm{p}<0.05)$.

Key words: severe mechanical injury, glucocorticoid osteoporosis, salmon calcitonin.

\section{ЛИТЕРАТУРА}

1. Беневоленская Л.И. Проблема остеопороза в современной медицине. Научно-практ. ревматол. 2005; 1: 4-7.

2. Буфистова А.В., Золотухин С.Е., Коробов В.П. [и др.] Прогнозирование тяжести течения глюкокортикоидного остеопороза (экспериментальное исследование). Загальна патологічна фізіологія. 2006; 1 (2): 60-64.

3. Ельский В.Н., Климовицкий В.Г., Золотухин С.Е. [и др.] Избранные аспекты патогенеза и лечения травматической болезни. Донецк: ООО «Лебедь», 2002. 360.

4. Золотухин C.Е. Травматическая болезнь у шахтеров глубоких угольных шахт. Клин. хир. 1998; 10: 33-36.

5. Казимирко В.К., Малышев В.И. Остеопороз как биологическая проблема. Здоров’я України. 2005; 21 (130): $27-29$.

6. Рощин Г.Г., Малыш Н.Р., Шлапак И.П., Сахно О.В. Прогностические критерии развития мультиорганной дисфункции у пострадавших с политравмой. Український журнал екстремальної медицини ім. Г.О. Можаєва. 2008; 9 (4): 27-32.

7. Chesnut C., Silverman S., Andriano K. [et al.]. A randomized trial of nasal spray salmon calcitonin in postmenopausal women with established osteoporosis: the prevent recurrence of osteoporotic fractures study. PROOF study group. Am J Med. 2000; 109 (4): 267-276.

8. Silverman SL, Azria M. The analgesic role of calcitonin following osteoporotic fracture. Osteoporos Int. 2002; 13 (11): 858-867. doi: $10.1007 / \mathrm{s} 001980200118$

\section{REFERENCES}

1. Benevolenskaya L.I. Problema osteoporoza v sovremennoi meditsine. Nauchno-prakt. revmatol. 2005; 1: 4-7 (in Russian).

2. Bufistova A.V., Zolotukhin S.E., Korobov V.P. [i dr.] Prognozirovanie tyazhesti techeniya glyukokortikoidnogo osteoporoza (eksperimental'noe issledovanie). Zagal'na patologichna fiziologiya. 2006; 1 (2): 60-64 (in Russian).

3. El'skii V.N., Klimovitskii V.G., Zolotukhin S.E. i dr. Izbrannye aspekty patogeneza i lecheniya travmaticheskoi bolezni. Donetsk: OOO «Lebed'», 2002. 360 (in Russian).

4. Zolotukhin S.E. Travmaticheskaya bolezn' u shakhterov glubokikh ugol'nykh shakht. Klin. khir. 1998; 10:33-36 (in Russian).

5. Kazimirko V.K., Malyshev V.I. Osteoporoz kak biologicheskaya problema. Zdorov'ya Ukraïni. 2005; 21 (130): 27-29 (in Russian).

6. G.G. Roshchin, N.R. Malysh, I.P. Shlapak, O.V. Sakhno. Prognosticheskie kriterii razvitiya mul'tiorgannoi disfunktsii u postradavshikh s politravmoi. Ukraïns'kii zhurnal ekstremal'noï meditsini im. G.O. Mozha€va. 2008; 9 (4): 27-32 (in Russian).

7. C. Chesnut, S. Silverman, K. Andriano [et al.]. A randomized trial of nasal spray salmon calcitonin in postmenopausal women with established osteoporosis: the prevent recurrence of osteoporotic fractures study. PROOF study group. Am J Med. 2000; 109 (4): 267-276.

8. Silverman SL, Azria M. The analgesic role of calcitonin following osteoporotic fracture. Osteoporos Int. 2002; 13 (11): 858-867. doi: 10.1007/s001980200118 